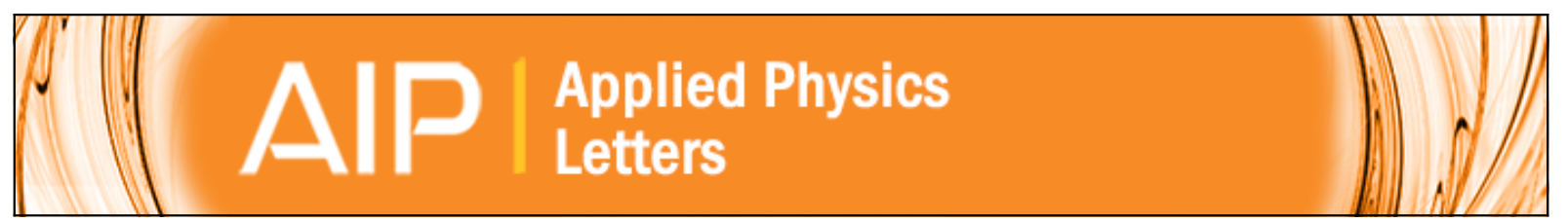

Band structure engineering and vacancy induced metallicity at the GaAs-AIAs interface M. Upadhyay Kahaly, S. Nazir, and U. Schwingenschlögl

Citation: Applied Physics Letters 99, 123501 (2011); doi: 10.1063/1.3643049

View online: http://dx.doi.org/10.1063/1.3643049

View Table of Contents: http://scitation.aip.org/content/aip/journal/apl/99/12?ver=pdfcov

Published by the AIP Publishing 


\title{
Band structure engineering and vacancy induced metallicity at the GaAs-AIAs interface
}

\author{
M. Upadhyay Kahaly, ${ }^{\text {a) }}$ S. Nazir, and U. Schwingenschlögl ${ }^{\text {b) }}$ \\ KAUST, Physical Science and Engineering, Thuwal 23955-6900, Kingdom of Saudi Arabia
}

(Received 21 June 2011; accepted 31 August 2011; published online 20 September 2011)

\begin{abstract}
We study the epitaxial GaAs-AlAs interface of wide gap materials by full-potential density functional theory. AlAs thin films on a GaAs substrate and GaAs thin films on an AlAs substrate show different trends for the electronic band gap with increasing film thickness. In both cases, we find an insulating state at the interface and a negligible charge transfer even after relaxation. Differences in the valence and conduction band edges suggest that the energy band discontinuities depend on the growth sequence. Introduction of As vacancies near the interface induces metallicity, which opens great potential for GaAs-AlAs heterostructures in modern electronics. (C) 2011 American Institute of Physics. [doi:10.1063/1.3643049]
\end{abstract}

Arsenide heterostructures (HSs) are commercially important ${ }^{1-5}$ with wide ranging applications in devices for optoelectronics, sensing, and lasers, for instance. The performance of these structures depends on the electronic properties of the substrate and the grown film. ${ }^{6}$ For example, the existence of two band gaps enables increased absorption of the broad solar spectrum in photovoltaic cells. However, a large lattice mismatch results in the generation of dislocations at the interface (IF), which in turn reduces the device efficiency. Hence, arsenide IFs formed of GaAs and AlAs, which have a very similar crystal lattice, are of interest since a long time. Many experimental investigations have been reported for various $(\mathrm{GaAs})_{m} /(\mathrm{AlAs})_{n}$ HSs with $(m+n)$ ranging from 2 to 10 , revealing unusual and exciting physical properties related to luminescence and optical absorption, ${ }^{7}$ two-photon absorption, ${ }^{8}$ and Raman as well as infrared spectra. ${ }^{9}$

Recently, it has been demonstrated experimentally that a bias voltage applied to a weakly coupled n-doped GaAs/ AlAs superlattice (SL) increases the amplitude of the hypersound oscillations generated by a femtosecond pulse. ${ }^{2}$ An anionic vacancy (a common form of defect introduced during realistic large scale synthesis) can result in n-doping. Hence, it is important to understand the effects of such vacancies on the heterointerface. Do they create, for example, similar effects as the $\mathrm{O}$ vacancies in the perovskite oxide HSs? ${ }^{10}$ Does a quasi two-dimensional conducting electron gas form at the IF? ${ }^{11}$ We attempt to address these issues in the present work by studying HSs formed by (i) AlAs thin films on a GaAs substrate and (ii) GaAs thin films on an AlAs substrate. After an introduction into the bulk crystal structures and structures of the $(\mathrm{GaAs})_{m} /(\mathrm{AlAs})_{n}$ and $(\mathrm{AlAs})_{m} /(\mathrm{GaAs})_{n}$ IFs, we will address our methodology and computational details. We then will discuss the possibility of critical thicknesses of the GaAs and AlAs layers as well as the IF relaxation. The electronic structures of various IFs are analyzed with a focus on the effect of As vacancies at or near the IF.

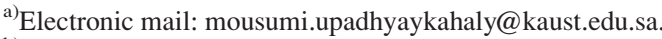

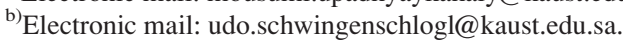

For studying the influence of the IF on the overall electronic properties of the HS, we address a single GaAs-AlAs IF in contrast to two or more IFs in quantum wells. We exclude the effect of strain by using lattice-matched structures and, for now, do not consider the effect of intersite chemical disorder (exchange of $\mathrm{Ga}$ and $\mathrm{Al}$ ), which can influence the defect-induced IF metallicity via an additional dipole contribution.

The calculations are performed using the full-potential linearized augmented plane wave plus local orbitals (FPLAPW + lo) method, as implemented in the Wien2k code, ${ }^{12}$ within the framework of density functional theory. For the self-consistent calculations, a $12 \times 12 \times 1 \mathrm{k}$-space grid with 28 points in the irreducible wedge of the Brillouin zone is employed. Relativistic effects are taken into account for the core states, while the scalar relativistic approximation is used for the valence states. The exchange-correlation contribution to the electron-electron interaction is modeled both by the local density approximation (LDA) and the generalized gradient approximation (GGA). For the wave function expansion inside the atomic spheres, a maximum value of $\ell_{\max }=12$ is used, together with a plane-wave cutoff of $R_{\operatorname{mt}} K_{\max }=7.0, G_{\max }=24$. Our basis set consists of the Ga $3 d^{10}, 4 s^{2}, 4 p^{1}, \mathrm{Al} 2 p^{6}, 3 s^{2}, 3 p^{1}$, and As $3 d^{10}, 4 s^{2}, 4 p^{3}$ states. The muffin-tin sphere radii $R_{m t}$ (in atomic units) are chosen to be 2.50 for both $\mathrm{Ga}$ and $\mathrm{Al}$, and 1.72 for As. All these parameters have been checked carefully for convergence to ensure correct results. In order to capture the effect of structural relaxation on the local chemical bonding and resulting physical properties of the IF, we have fully optimized the HSs by relaxing the atomic forces to less than $5 \mathrm{mRy} / \mathrm{a}$.u.

Both $\mathrm{GaAs}$ and AlAs crystallize in the zinc-blende structure with space group no. $216(F \overline{4} 3 m)$. They are insulators with experimental band gaps of $1.41 \mathrm{eV}$ (Ref. 13) and $2.23 \mathrm{eV},{ }^{14}$ respectively. The lattice constants are $5.65 \AA$ for GaAs and $5.66 \AA$ for AlAs. ${ }^{13,14}$ Owing to the almost perfect matching of the lattice constants (lattice mismatch $<1 \%$ ), GaAs/AlAs HSs exhibit minor strain effects. This suggests that, at least in a first-order approach, one can neglect the lattice distortion and assume that the atomic positions of the anion and the cation sublattices remain unchanged from the 


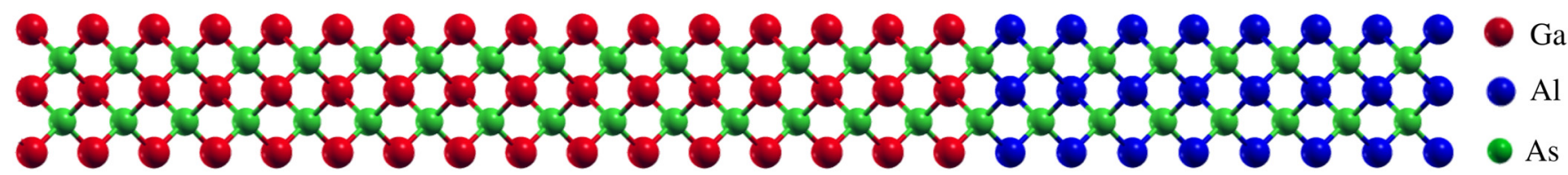

FIG. 1. (Color online) Schematic representation of the $(\mathrm{GaAs})_{8} /(\mathrm{AlAs})_{n} \mathrm{HS}$, with $n=4$. The IF is As terminated.

bulk to the HSs. However, we note that despite an identical average composition the properties of HSs can differ depending on the particular ordering of the cation sublattice.

We first study the electronic structure of the bulk compounds. The band gaps of GaAs calculated by the LDA and GGA are $0.28 \mathrm{eV}$ and $0.51 \mathrm{eV}$, respectively, which agree well with previous reports. ${ }^{15}$ For AlAs, the LDA and GGA energy gaps are found to be $1.35 \mathrm{eV}$ and $1.43 \mathrm{eV}$, respectively, which are also in good agreement with previous calculations. ${ }^{16}$ Since there is a remarkable difference between the LDA and GGA results, and the GGA band gap is closer to the experimental value, we use only the GGA in the IF study. In order to model the HSs, the supercell approach is used with the average lattice constant of GaAs and AlAs (5.655 $\AA$ ). We study GaAs and AlAs substrates which both are terminated by the As layer. First, in order to check for a critical thickness of the thin film slabs in both these HSs to impart conductivity, we change the thickness of the thin film from 1 to 4 unit cells for a substrate thickness of $4.51 \mathrm{~nm}(8$ unit cells). The structural setup is illustrated in Fig. 1.

For both configurations, the valence bands show almost equal contributions from the $\mathrm{Ga} / \mathrm{Al}$ and $\mathrm{As} s$ and $p$ states (see Figs. 2 and 3). This indicates a covalent nature of the IFs. The low-lying $d$ states are not shown. For configuration (i), the valence band edges, for all $n=1-4$, remain just below the Fermi energy $\left(E_{F}\right)$, while the conduction band edge shifts closer to $E_{F}$, though very slowly, with increasing thickness of the AlAs film. The conduction bands exhibit major contributions from the $\mathrm{Al}$ and $\mathrm{Ga}$ states, with partial As contributions. Configuration (ii) shows similar features in the electronic structure. The slow change of the band gap (Figs. 2 and 3)

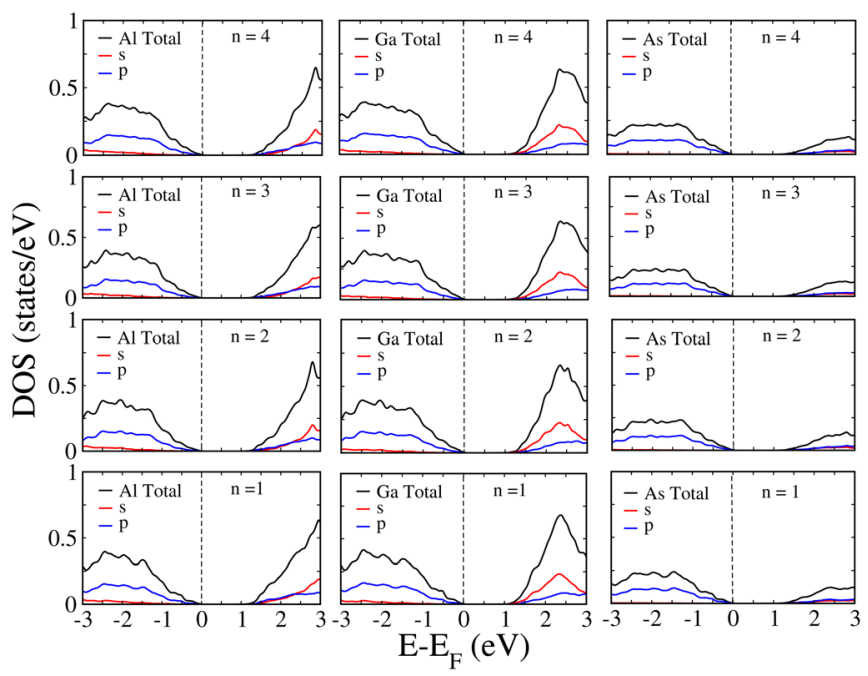

FIG. 2. (Color online) Effect of the AlAs thin film thickness on the projected DOS of the Al (first column), Ga (second column), and As (third column) atoms nearest to the IF of the $(\mathrm{GaAs})_{8} /(\mathrm{AlAs})_{n} \mathrm{HS}$, with $n$ varying from 1 (bottom row) to 4 (top row). The dotted line in each panel represents $E_{F}$. with the thickness of the film for all systems under investigation suggests that there is no critical IF thickness. Figs. 2 and 3 show that the two isoelectronic cations $\mathrm{Al}$ and $\mathrm{Ga}$ contribute similarly to the electronic structures of the two HSs.

It is known that IF states depend critically on the structural details in oxide HSs. ${ }^{17,18}$ For this reason, we take into account the structural relaxation in our calculations. However, due to the minimal lattice mismatch between the component materials, the structure optimization results only in negligible changes $(<0.0005 \AA)$ of the bond lengths near the IF. The electronic states of both configuration remain virtually the same after the relaxation. Thus, a charge transfer induced by the IF relaxation plays no role for the conductivity of the systems under investigation.

To study the effect of As vacancies, we use a $1 \times 2 \times 12$ supercell which consists of 8 layers of GaAs and 4 layers of AlAs. A corresponding setup is considered for the alternative HS as well. First, we introduce $25 \%$ of As deficiency in the IF layer. Second, As deficiency in the layer above or below the IF is analysed. The calculated DOSs depicted in Figs. 4 and 5 clearly indicate an induced metallicity for all the As vacancy locations. The metallic states are determined by (almost equal) contributions from the isoelectronic cations, $\mathrm{Ga}$ and $\mathrm{Al} . \mathrm{As}^{2+}$ has an $4 s^{2} 4 p^{3}$ electronic configuration that is essential to form the $s p^{3}$ bond with the neighbouring group-III cations. When an As vacancy is created, the broken $s p^{3}$ hybrid bond provides extra electrons to the $\mathrm{Ga} / \mathrm{Al} s$ and $p$ orbitals and fills them partially. As a consequence, these states shift considerably to lower energy, crossing the $E_{F}$ and leave the IF metallic. While the valence bands exhibit major contributions from the $\mathrm{Ga} / \mathrm{Al} p$ states, the conduction bands mainly originate from the As $p$ orbitals, see Figs. 4 and 5.

Interestingly, the electronic DOS at $E_{F}$ remains almost equal for any location of the As vacancy (see Figs. 4 and 5),

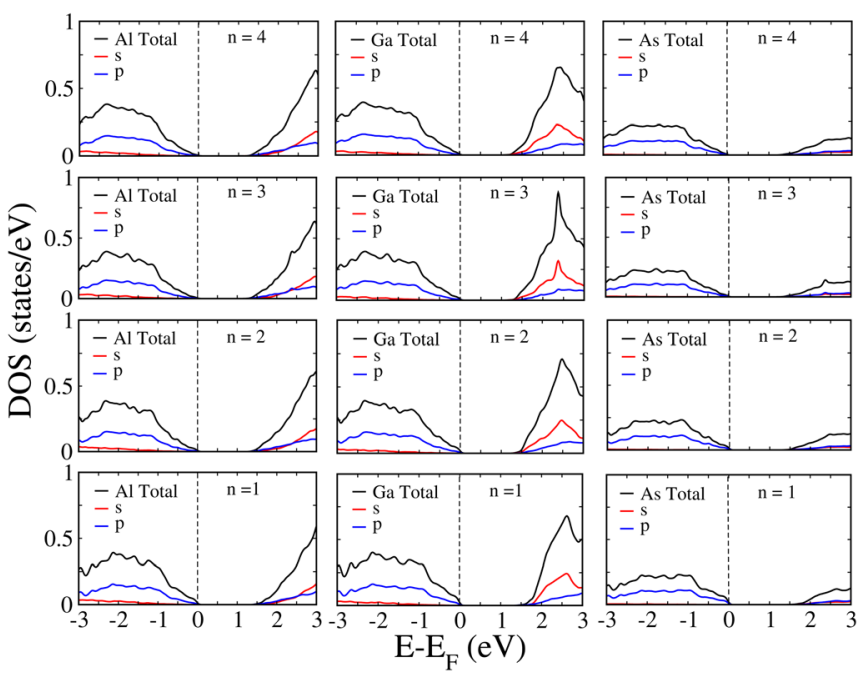

FIG. 3. (Color online) Analogous to Fig. 2 but for the (AlAs) $)_{8} /(\mathrm{GaAs})_{n} \mathrm{HS}$. 


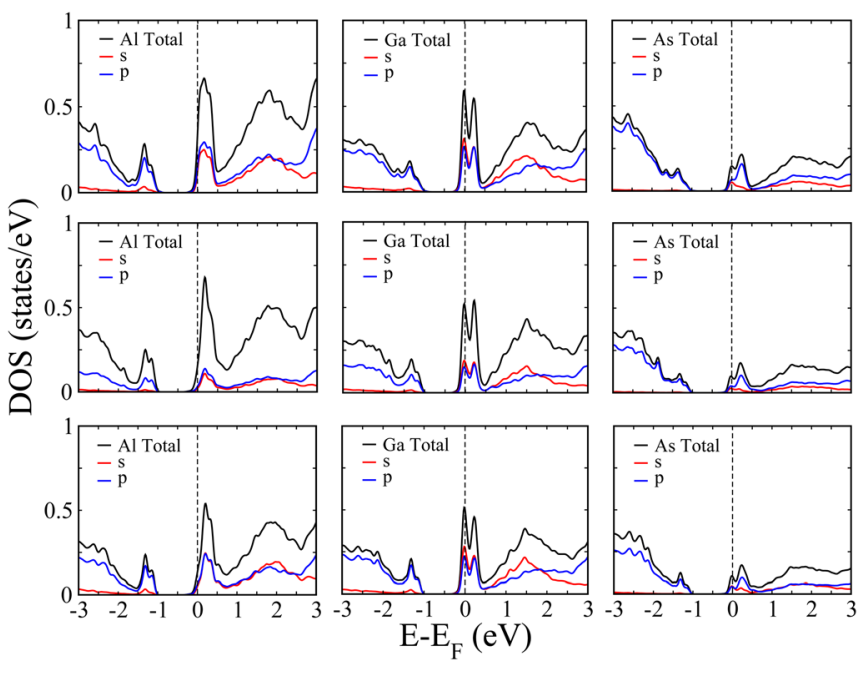

FIG. 4. (Color online) $(\mathrm{GaAs})_{8} /(\mathrm{AlAs})_{4} \mathrm{IF}$ : As vacancy induced projected DOS of the Al (first column), Ga (second column), and As (third column) atoms. While the central row is for an As vacancy created at the IF, the top and bottom rows are for As vacancies one layer above and below the IF, respectively. The dotted line in each panel represents $E_{F}$.

indicating that the induced metallicity is indeed intrinsic property to the As deficient HS. The conduction band DOS spectra demonstrate that the As deficient IFs have a freeelectron like nature, which is highly relevant for technological applications. Since As vacancies act as an $n$-type dopants, $E_{F}$ is shifted to higher energy and crosses the previous conduction band edge, compare Fig. 2 to Fig. 4 . This makes the available electrons at the IF highly mobile, creating an electron gas. We have also investigated a vacancy concentration of $12.5 \%$ and find the metallic state to persist. Comparison to the results for $25 \%$ concentration suggests that the metallic state will persist even for lower concentration. We find the

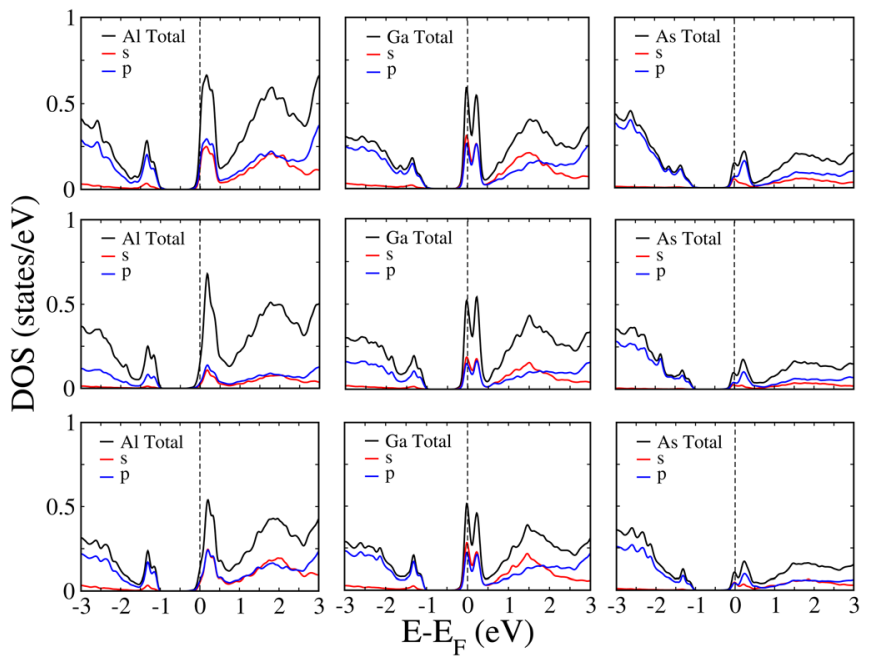

FIG. 5. (Color online) Analogous to Fig. 4 but for the (AlAs $)_{8} /(\mathrm{GaAs})_{4} \mathrm{IF}$. thickness of the conduction zone to be $10-12 \AA$, depending on the location of the As vacancy. This fact confirms the quasi two-dimensional nature of the vacancy-induced electron gas. The conduction band peak just above the $E_{F}$ becomes sharper when the As vacancy is located further away from the IF, which reflects the tendency to form a conduction band edge in this situation. A similar behavior is found for configuration (ii). Therefore, we conclude for both HSs that the conductivity is a result of As defects and, consequently, can be controlled by the As concentration.

In conclusion, we have studied the electronic properties of the GaAs-AlAs heterointerfaces using all electron band structure calculations based on density functional theory. We find an insulating state if the system is stoichiometric, which hardly changes for increasing thicknesses of the AlAs and GaAs layers (both with and without consideration of the structure relaxation). However, it turns out that As vacancies at and near the IF lead to a conducting quasi twodimensional electron gas between the two insulating dielectric arsenides. The metallicity is expected to be enhanced with growing As vacancy concentration, irrespective of the exact location of the As defects. Therefore, this n-doped heterointerface has great potential in applications in nanoelectronic arsenide-based devices.

${ }^{1}$ T. Kitada, F. Tanaka, T. Takahashi, K. Morita, and T. Isu, Appl. Phys. Lett. 95, 111106 (2009).

${ }^{2}$ R. P. Beardsley, A. V. Akimov, M. Henini, and A. J. Kent, Phys. Rev. Lett. 104, 085501 (2010).

${ }^{3}$ D. Hu, C. C. McPheeters, E. T. Yu, and D. M. Schaadt, Nanoscale Res. Lett. 6, 83 (2011).

${ }^{4}$ A. J. Kent, R. N. Kini, N. M. Stanton, M. Henini, B. A. Glavin, V. A. Kochelap, and T. L. Linnik, Phys. Rev. Lett. 96, 215504 (2006).

${ }^{5}$ R. N. Kini, A. J. Kent, N. M. Stanton, and M. Henini, J. Appl. Phys. 98, 033514 (2005).

${ }^{6}$ G. Mariani, P. Wong, A. M. Katzenmeyer, F. Lonard, J. Shapiro, and D. L. Huffaker, Nano Lett. 11, 2490 (2011).

${ }^{7}$ A. C. Gossard, P. M. Petroff, W. Wiegmann, R. Dingle, and A. Savage, Appl. Phys. Lett. 29, 323 (1976).

${ }^{8}$ J. P. van der Ziel and A. C. Gossard, Phys. Rev. B 17, 765 (1978).

${ }^{9}$ A. S. Barker, Jr., J. L. Merz, and A. C. Gossard, Phys. Rev. B 17, 3181 (1978).

${ }^{10}$ R. Pentcheva and W. E. Pickett, Phys. Rev. B 74, 035112 (2006).

${ }^{11}$ S. Thiel, G. Hammerl, A. Schmehl, C. W. Schneider, and J. Mannhart, Science 313, 1942 (2006).

${ }^{12}$ P. Blaha, K. Schwarz, G. Madsen, D. Kvasicka, and J. Luitz, WIEN2k. An augmented plane wave + local orbitals program for calculating crystal properties, TU Vienna, Vienna, 2001.

${ }^{13}$ Semiconductors, Group IV Elements and III-V Compounds, Landolt-Bornstein, New Series, Group III, Vol. 17, edited by O. Madelung (Springer, Berlin, 1991).

${ }^{14}$ B. Monemar, Phys. Rev. B 8, 5711 (1973).

${ }^{15}$ H. Arabia, A. Pourghazi, F. Ahmadian, and Z. Nourbakhsh, Physica B 373, 16 (2006).

${ }^{16}$ H. Jin, G. L. Zhao, and D. Bagayoko, Phys. Rev. B 73, 245214 (2006).

${ }^{17}$ U. Schwingenschlögl and C. Schuster, EPL 86, 27005 (2009).

${ }^{18}$ S. Nazir and U. Schwingenschlögl, Appl. Phys. Lett 99, 073120 (2011). 